\title{
M/G/1 Retrial Queue with Priority, Collisions and Feedback Customers
}

\author{
M.Nila ${ }^{1}$, Dr. D. Sumitha ${ }^{2}$ \\ \{ brightmoon02@gmail.com ${ }^{1}$, sumitha4677@gmail.com ${ }^{2}$ \}

\begin{abstract}
Research Scholar, Department of Mathematics, Avinashilingam Institute for Home science and
Associate Professor, Department of Science and Humanities, Avinashilingam Institute for Home science and Higher Education for Women, Coimbatore, Tamil Nadu, India. ${ }^{2}$
\end{abstract} \\ Higher Education for Women, Coimbatore, Tamil Nadu, India ${ }^{1}$
}

\begin{abstract}
This articles provides a detailed study of retrial queueing model with collisions, feedback and priority customers. The motive for this model come from various application in packet switching networks, random access protocols in local area networks, e-commerce systems, production systems and real time situations. The objective of the work is to derive the performance measures like stable state probabilities, regular systems extent and regular orbit size of the proposed model by Supplementary variable Method. Accept that customer attain the systems conferring to Poisson procedure. If the servers is demanding the incoming consignment either enter to the retrial queue or one of the customer disrupts the customers in facility to get his service and the disrupted customer with remaining customers join the retrial queue or creates a collision with existing customer and all being shifted to the retrial queue. When the server is free, the customer in the incoming batch begins the service directly and breaks change to the path. The customer is allowed to make a feedback. We discuss special cases for this model and we analyse the numerical results of effects in various limits on the system presentation actions.
\end{abstract}

Keywords: Batch arrival, Retrial queue, Pre-emptive resume, priority, Collisions, Feedback, Orbital search.

\section{Introduction}

Retrial queues are identify by the detail that a consumer alighting when all accessible server are earnest leave the facilities area but afterward sometime repeat the adjure. This feature plays a vital role in telecommunication networks, cognitive networks, cloud computing systems, production, manufacturing systems etc. The incentive for learning a batch entrance retrial queueing system comes from some requests faced in call centers, optical burst switching networks, CSMA protocols etc. The present paper deals with lot advent retrial queueing model with Pre-emptive recommence importance, Collisions, Orbital and feedback Search.

The articles is prearranged as tracks. The survey of the earlier pertinent works is given in segment II. In section III, we analyse the prescribed model. The steady state distributions and solutions are deliberated in section IV. In section V, Performance measures are derived. Special cases are discussed in section VI. Section VII, Stochastic rottenness law has been verified for this model. Section VIII, explores the numerical results for this model. 


\section{Literature Survey}

Precedence queues, together non-pre-emptive and pre-emptive have critical uses inside the modelling and exploration of computers system, verbal exchange network, production models, running systems and are broadly utilized in production exercise and transportation control. carrier priority is sincerely nowadays a main function of any production machine. Many authors have mentioned pre-emptive resume precedence queueing structures. Krishna Kumar et al. (2002) taken into consideration queueing machine with two segment service and pre-emptive resume. Jain and Charu Bhargava (2008) studied bulk arrival retrial queue with untrustworthy server and precedence subscriber. Chen and Zhu (2010) dealt retrial queue with importance, balking and response customer. Dimitriou (2013) illustrated pre-emptive resume precedence retrial queue with national recognized influxes, untrustworthy serverand terrible clients. Peng (2016) illustrated discrete time retrial queueing gadget with preemptive resume and Bernoulli feedback. Ammar and Rajadurai (2019) analyzed overall performance measures of pre-emptive precedence retrial queueing machine with disaster underneath running failure service.

Retrial queue with collision are commendable for modeling the procedure in communique community, nearby location network with institution allocation and a couple of get right of entry to protocol. Krishna Kumar et al. (2010) discussed single servers feed-back retrial queue with collision. Tao et al. (2012) examined M/M/1 retrial queue with collision and running vacation interruption under $\mathrm{N}$ coverage. Liu et al. (2014) investigated retrial G-queue with pre-emptive resume precedence and collision subjects to the server breakdown and behind schedule repair. Kvach and Nazaro (2015) mentioned markovian retrial machine with collisions. Toth et al. (2017) considered retrial queue with non reliable server and collisions. Tong et al. (2019) dealt retrial queue with working excursion and collision.

\section{Model Description}

In this segment, we contemplate consignment arrival retrial queue with pre-emptive resume importance, collision, response and detour search. Customer reach at the systems in accordance with Poisson stream of rate $\lambda$. If $\mathrm{Y}$ is a random capricious, then batch size distribution is given byP $\{\mathrm{Y}=\mathrm{k}\}=\mathrm{C}_{\mathrm{k}}, \mathrm{k}=1,2,3 \ldots,{ }_{\mathrm{k}=1}^{\infty} \mathrm{C}_{\mathrm{k}}=1$, the possibility generating function (PGF) $\mathrm{C}(\mathrm{z}$ ) having dual instants $\mathrm{m} 1$ and $\mathrm{m} 2$. If an arriving consignment discoveries the server indolent, one of the clients from the batch gets the carrier proximately, and the relaxation input into the retrial queue and try again subsequently a few random quantity of time. The cumulative distribution characteristic (cdf), opportunity density characteristic (pdf), Laplace Stieltjes transform (LST) of retrial time are represented by way of $\mathrm{A}(\mathrm{x}), \mathrm{a}(\mathrm{x}), \mathrm{A} *(\mathrm{~s})$ respectively.

on the other hand, if the servers is busy, then the arrival batch proceed to the servers with possibility $\tau$. when the incoming batch proceeds to the server, one of the purchaser inside the batch both disrupts with the patron in provider to get his personal service with opportunity $\kappa$ 
and the disrupted customer at the side of others arrive into the retrial queue or crash with the consumer in service resulting in each being transferred to the retrial queue in conjunction with arriving batch with complementary opportunity. The cdf, pdf and LST of service time are represented with the aid of $\mathrm{B}(\mathrm{x}), \mathrm{b}(\mathrm{x}), \mathrm{B}^{*}(\mathrm{~s})$ respectively with first moments $\mu 1$ and $\mu 2$. After executing provider, the purchaser may go returned to the retrial queue as a comments consumer for purchasing another provider with chance $\delta$ or departs the machine. On each service crowning glory the server takes clients from the retrial queue for carrier with possibility $\theta$ or stays idle.

$$
\eta(x)=a(x) / 1-A(x) \text { and } \mu(x)=b(x) / 1-B(x) \text {. }
$$

\section{Steady state Distribution}

The Steady state equation leading the model are given below

$$
\begin{aligned}
& \lambda \mathrm{R}_{0}=\bar{\delta} \int_{0}^{\infty} S_{0}(\mathrm{x}) \mu(\mathrm{x}) \mathrm{dx} \\
& \frac{\mathrm{d}}{\mathrm{dx}} \mathrm{R}_{\mathrm{n}}(\mathrm{x})=-[\lambda+\eta(\mathrm{x})] \mathrm{R}_{\mathrm{n}}(\mathrm{x}), \mathrm{n} \geq 1 \\
& \frac{\mathrm{d}}{\mathrm{dx}} S_{\mathrm{n}}(\mathrm{x})=-[\lambda+\mu(\mathrm{x})] S_{\mathrm{n}}(\mathrm{x})+(1-\tau) \lambda \sum_{k=1}^{n} \mathrm{C}_{\mathrm{k}} S_{\mathrm{n}-\mathrm{k}}(\mathrm{x}) \\
& \mathrm{n} \geq 0
\end{aligned}
$$

With boundary condition

$$
\begin{gathered}
\mathrm{R}_{1}(0)=\bar{\theta} \bar{\delta} \int_{0}^{\infty} \mathrm{S}_{1}(\mathrm{x}) \mu(\mathrm{x}) \mathrm{dx}+\bar{\theta} \delta \int_{0}^{\infty} \mathrm{S}_{0}(\mathrm{x}) \mu(\mathrm{x}) \mathrm{dx} \\
\mathrm{R}_{\mathrm{n}}(0)=\bar{\theta} \bar{\delta} \int_{0}^{\infty} \mathrm{S}_{\mathrm{n}}(\mathrm{x}) \mu(\mathrm{x}) \mathrm{d} \mathrm{x}+\bar{\theta} \delta \int_{0}^{\infty} \mathrm{S}_{\mathrm{n}-1}(\mathrm{x}) \mu(\mathrm{x}) \mathrm{d} \mathrm{x}+ \\
\overline{\mathrm{\kappa}} \tau \lambda \int_{0}^{\infty} \sum_{k=1}^{n} \mathrm{C}_{\mathrm{K}} \mathrm{S}_{\mathrm{n}-(\mathrm{k}+1)}(\mathrm{x}) \mathrm{dx}, \mathrm{n} \geq 2 \\
S_{0}(0)=\lambda \mathrm{c}_{1} \mathrm{R}_{0}+\int_{0}^{\infty} \mathrm{R}_{1}(\mathrm{x}) \eta(\mathrm{x}) \mathrm{dx}+\theta \bar{\delta} \int_{0}^{\infty} \mathrm{S}_{1}(\mathrm{x}) \mu(\mathrm{x}) \mathrm{dx} \\
+\theta \delta \int_{0}^{\infty} S_{0}(\mathrm{x}) \mu(\mathrm{x}) \mathrm{dx} \\
\mathrm{S}_{\mathrm{n}}(0)=\tau \lambda \kappa \int_{0}^{\infty} \sum_{k=1}^{n} \mathrm{C}_{\mathrm{K}} S_{\mathrm{n}-\mathrm{k}}(\mathrm{x}) \mathrm{dx}+\lambda \mathrm{c}_{\mathrm{n}+1} R_{0}+ \\
\int_{0}^{\infty} R_{\mathrm{n}+1}(\mathrm{x}) \eta(\mathrm{x}) \mathrm{dx}+\quad \lambda \int_{0}^{\infty} \sum_{k=1}^{n} \mathrm{C}_{\mathrm{K}} R_{\mathrm{n}-\mathrm{k}+1}(\mathrm{x}) \mathrm{dx}+ \\
\theta \bar{\delta} \int_{0}^{\infty} S_{\mathrm{n}+1}(\mathrm{x}) \mu(\mathrm{x}) \mathrm{dx}+\theta \delta \int_{0}^{\infty} S_{\mathrm{n}}(\mathrm{x}) \mu(\mathrm{x}) \mathrm{dx}
\end{gathered}
$$

The normalizing conditions is 


$$
R_{0}+\sum_{n=1}^{\infty} \int_{0}^{\infty} R_{\mathrm{n}}(\mathrm{x}) \mathrm{dx}+\sum_{n=0}^{\infty} \int_{0}^{\infty} S_{\mathrm{n}}(\mathrm{x}) \mathrm{dx}=1
$$

To solve these equations define the probability making function as

$$
\mathrm{R}(\mathrm{x}, \mathrm{z})=\sum_{n=1}^{\infty} \mathrm{R}_{\mathrm{n}}(\mathrm{x}) \mathrm{z}^{\mathrm{n}}, \mathrm{S}(\mathrm{x}, \mathrm{z})=\sum_{n=0}^{\infty} S_{\mathrm{n}}(\mathrm{x}) \mathrm{z}^{\mathrm{n}}
$$

Multiplying equations (1) - (7) by $\mathrm{z}^{\mathrm{n}}$ and solving we have the following equation

$$
\begin{aligned}
& \left(\frac{\partial}{\partial x}+\lambda+\eta(\mathrm{x})\right) R(\mathrm{x}, \mathrm{z})=0 \\
& \left(\frac{\partial}{\partial x}+\lambda(1-\bar{\tau} \mathrm{c}(\mathrm{z}))+\mu(\mathrm{x})\right) S(\mathrm{x}, \mathrm{z})=0 \\
& \mathrm{R}(0, \mathrm{z})=(\bar{\theta} \bar{\delta}+\bar{\theta} \delta \mathrm{z}) \int_{0}^{\infty} S(\mathrm{x}, \mathrm{z}) \mu(\mathrm{x}) \mathrm{dx}+ \\
& \quad \lambda \tau \overline{\mathrm{K}} \mathrm{zc}(\mathrm{z}) \int_{0}^{\infty} S(\mathrm{x}, \mathrm{z}) \mathrm{dx}-\lambda R_{0} \\
& \mathrm{~S}(0, \mathrm{z})=\frac{\lambda c(\mathrm{z}) R_{0}}{\mathrm{z}}+\frac{1}{\mathrm{z}} \int_{0}^{\infty} R(\mathrm{x}, \mathrm{z}) \eta(\mathrm{x}) \mathrm{dx}+\frac{\lambda c(\mathrm{z})}{\mathrm{z}} \int_{0}^{\infty} R(\mathrm{x}, \mathrm{z}) \mathrm{dx} \\
& +\left(\frac{\delta \theta \mathrm{z}+\theta \bar{\delta}}{\mathrm{z}}\right) \int_{0}^{\infty} S(\mathrm{x}, \mathrm{z}) \mu(\mathrm{x}) \mathrm{d} \mathrm{x}+\kappa \tau \lambda c(\mathrm{z}) \int_{0}^{\infty} S(\mathrm{x}, \mathrm{z}) \mathrm{dx}
\end{aligned}
$$

Solving the partial differential equations (9) - (12),

$$
\begin{gathered}
\mathrm{R}(\mathrm{x}, \mathrm{z})=\mathrm{R}(0, \mathrm{z}) \mathrm{e}^{-\lambda \mathrm{x}}(1-\mathrm{A}(\mathrm{x})) \\
\mathrm{S}(\mathrm{x}, \mathrm{z})=\mathrm{S}(0, \mathrm{z}) \mathrm{e}^{-\lambda[1-\overline{\mathrm{\tau}} \mathrm{c}(\mathrm{z})] \mathrm{x}}(1-\mathrm{B}(\mathrm{x}))
\end{gathered}
$$

Using the equations (13 ) - (14) in (11) - (12) we obtain

$$
\begin{aligned}
& R(0, \mathrm{z})=\lambda R_{0}\{(1-\bar{\tau} \mathrm{c}(\mathrm{z}))[\mathrm{z}-\mathrm{c}(\mathrm{z}) \bar{\theta}(\bar{\delta}+\delta \mathrm{z}) \\
& \left.\mathrm{B}^{*}(\lambda-\bar{\tau} \lambda \mathrm{c}(\mathrm{z}))-\theta(\bar{\delta}+\delta \mathrm{z}) \mathrm{B}^{*}(\lambda-\bar{\tau} \lambda \mathrm{c}(\mathrm{z}))\right]-\mathrm{\tau zc}(\mathrm{z}) \\
& \left.\left(1-\mathrm{B}^{*}(\lambda-\bar{\tau} \lambda \mathrm{c}(\mathrm{z}))\right)[\kappa+\bar{\kappa} \mathrm{c}(\mathrm{z})]\right\} / \mathrm{D}(\mathrm{z}) \\
& \mathrm{S}(0, \mathrm{z})=\lambda \mathrm{R}_{0}(1-\mathrm{c}(\mathrm{z})) \mathrm{A}^{*}(\lambda)(1-\bar{\tau} \mathrm{c}(\mathrm{z})) / \mathrm{D}(\mathrm{z}) \\
& \mathrm{D}(\mathrm{z})=(1-\bar{\tau} \mathrm{c}(\mathrm{z}))\left\{\left[\mathrm{c}(\mathrm{z})+(1-\mathrm{c}(\mathrm{z})) \mathrm{A}^{*}(\lambda)\right] \bar{\theta}(\bar{\delta}+\right. \\
& \left.\delta \mathrm{z}) \mathrm{B}^{*}(\lambda-\bar{\tau} \lambda \mathrm{c}(\mathrm{z}))+\theta(\bar{\delta}+\delta \mathrm{z}) \mathrm{B}^{*}(\lambda-\bar{\tau} \lambda \mathrm{c}(\mathrm{z}))-\mathrm{z}\right\}+ \\
& {\left[\mathrm{c}(\mathrm{z})+(1-\mathrm{c}(\mathrm{z})) \mathrm{A}^{*}(\lambda)\right] \tau \mathrm{zc}(\mathrm{z}) \bar{\kappa}\left(1-\mathrm{B}^{*}(\lambda-\right.} \\
& \bar{\tau} \lambda \mathrm{c}(\mathrm{z})))+\tau \mathrm{zc}(\mathrm{z}) \kappa\left(1-\mathrm{B}^{*}(\lambda-\bar{\tau} \lambda \mathrm{c}(\mathrm{z}))\right)
\end{aligned}
$$


Putting $\mathrm{R}(0, \mathrm{z})$ and $\mathrm{S}(0, \mathrm{z})$ in the expressions of $\mathrm{R}(\mathrm{x}, \mathrm{z})$ and $\mathrm{S}(\mathrm{x}, \mathrm{z})$ and integrating with admiration to $\mathrm{x}$, we get

$$
\begin{gathered}
R(\mathrm{z})=\mathrm{R}_{0}\left(1-\mathrm{A}^{*}(\lambda)\right)\{(1-\bar{\tau} \mathrm{c}(\mathrm{z}))[\mathrm{z}-\mathrm{c}(\mathrm{z}) \bar{\theta}(\bar{\delta}+ \\
\left.\delta \mathrm{z}) \mathrm{B}^{*}(\lambda-\bar{\tau} \lambda \mathrm{c}(\mathrm{z}))-\theta(\bar{\delta}+\delta \mathrm{z}) \mathrm{B}^{*}(\lambda-\bar{\tau} \lambda \mathrm{c}(\mathrm{z}))\right]- \\
\left.\tau \mathrm{zc}(\mathrm{z})\left(1-\mathrm{B}^{*}(\lambda-\bar{\tau} \lambda \mathrm{c}(\mathrm{z}))\right)[\kappa+\bar{\kappa} \mathrm{c}(\mathrm{z})]\right\} / \mathrm{D}(\mathrm{z}) \\
\mathrm{S}(\mathrm{z})=R_{0}(1-\mathrm{c}(\mathrm{z})) \mathrm{A}^{*}(\lambda)\left[1-\mathrm{B}^{*}(\lambda-\bar{\tau} \lambda \mathrm{c}(\mathrm{z}))\right] / \mathrm{D}(\mathrm{z})
\end{gathered}
$$

\section{Performance Measures}

The probability of the idle server during retrial time is

$$
\begin{aligned}
\mathrm{R}= & \left(1-\mathrm{A}^{*}(\lambda)\right) \mathrm{R}_{0}\left\{\left(1-\mathrm{B}^{*}(\tau \lambda)\right)\left[\tau+\mathrm{m}_{1}+\tau \mathrm{m}_{1} \overline{\mathrm{\kappa}}\right]-\right. \\
& \left.\tau\left[1-\delta \mathrm{B}^{*}(\tau \lambda)-\bar{\theta} \mathrm{m}_{1} \mathrm{~B}^{*}(\tau \lambda)\right]\right\} / \mathrm{T}_{1}
\end{aligned}
$$

The probability of the busy server is given by

$$
\begin{aligned}
\mathrm{S}=R_{0} \mathrm{~m}_{1} \mathrm{~A}^{*}(\lambda)\left[1-\mathrm{B}^{*}(\tau \lambda)\right] / \mathrm{T}_{1} \\
\mathrm{~T}_{1}=\tau \mathrm{B}^{*}(\tau \lambda) \bar{\delta}-\mathrm{m}_{1}\left(1-\mathrm{B}^{*}(\tau \lambda)\right)-\mathrm{m}_{1}\left(1-\mathrm{A}^{*}(\lambda)\right) \\
\quad\left[\tau \mathrm{B}^{*}(\tau \lambda) \bar{\theta}+\tau \bar{\kappa}\left(1-\mathrm{B}^{*}(\tau \lambda)\right)\right]
\end{aligned}
$$

Normalising condition (8) is equivalent to $R_{0}+R+S=1$, relieving the vocabularies of $R$, $\mathrm{S}$ we get

$$
R_{0}=\mathrm{T}_{1} / \tau \bar{\delta} \mathrm{A}^{*}(\lambda) \mathrm{B}^{*}(\tau \lambda)
$$

The PGF of system size is

$$
\begin{aligned}
\mathrm{P}_{\mathrm{s}}(\mathrm{z}) & =R_{0}+\mathrm{R}(\mathrm{z})+\mathrm{zS}(\mathrm{z}) \\
= & \mathrm{R}_{0} \mathrm{~A}^{*}(\lambda)(1-\bar{\tau} \mathrm{c}(\mathrm{z})) \mathrm{B}^{*}(\lambda-\bar{\tau} \lambda \mathrm{c}(\mathrm{z}))(1-\mathrm{z}) \bar{\delta} / \mathrm{D}(\mathrm{z})
\end{aligned}
$$

The PGF of orbit size is

$$
\begin{aligned}
\mathrm{P}_{\mathrm{q}}(\mathrm{z})= & R_{0}+\mathrm{R}(\mathrm{z})+\mathrm{S}(\mathrm{z}) \\
= & \mathrm{R}_{0} \mathrm{~A}^{*}(\lambda)\left\{(1-\bar{\tau} \mathrm{c}(\mathrm{z}))\left[\mathrm{B}^{*}(\lambda-\bar{\tau} \lambda \mathrm{c}(\mathrm{z}))(\delta \mathrm{z}+\bar{\delta})-\mathrm{z}\right]\right. \\
& \left.+\left(1-\mathrm{B}^{*}(\lambda-\bar{\tau} \lambda \mathrm{c}(\mathrm{z}))\right)[\mathrm{zc}(\mathrm{z}) \tau+1-\mathrm{c}(\mathrm{z})]\right\} / \mathrm{D}(\mathrm{z})
\end{aligned}
$$


The average orbit size is given by

$$
\mathrm{L}_{q}=\lim _{z \rightarrow 1} \frac{\mathrm{d}}{\mathrm{dz}} \mathrm{P}_{q}(\mathrm{z})=\frac{\mathrm{N}_{2} \mathrm{D}_{1}-\mathrm{D}_{2} \mathrm{~N}_{1}}{2\left(\mathrm{D}_{1}\right)^{2}}
$$

Where

$$
\begin{aligned}
\mathrm{N}_{1}=-\mathrm{I}_{0} \mathrm{~A}^{*}(\lambda) \tau \bar{\delta} \mathrm{B}^{*}(\tau \lambda) \\
\mathrm{N}_{2}=-2 \mathrm{I}_{0} \mathrm{~A}^{*}(\lambda)\left[\tau \bar{\delta} \mathrm{k}_{1}+\mathrm{m}_{1}(\tau+\delta \bar{\tau}) \mathrm{B}^{*}(\tau \lambda)+\mathrm{m}_{1}\right] \\
\mathrm{D}_{1}=\mathrm{m}_{1}\left(1-\mathrm{B}^{*}(\tau \lambda)\right)-\tau \mathrm{B}^{*}(\tau \lambda) \bar{\delta}+\mathrm{m}_{1}\left(1-\mathrm{A}^{*}(\lambda)\right) \\
\left.\qquad \tau \mathrm{B}^{*}(\tau \lambda) \bar{\theta}+\tau \bar{\kappa}\left(1-\mathrm{B}^{*}(\tau \lambda)\right)\right] \\
\mathrm{D}_{2}=\left(1-\mathrm{B}^{*}(\tau \lambda)\right)\left\{\mathrm{m}_{2}+\tau \overline{\mathrm{\kappa}}\left(1-\mathrm{A}^{*}(\lambda)\right)\left[\mathrm{m}_{2}+2 \mathrm{~m}_{1}\right.\right. \\
\left.\left.+2 \mathrm{~m}_{1}^{2}\right]\right\}+\tau \mathrm{m}_{2} \bar{\theta} \mathrm{B}^{*}(\tau \lambda)\left(1-\mathrm{A}^{*}(\lambda)\right)+2 \mathrm{~m}_{1}\left(1-\mathrm{A}^{*}(\lambda)\right) \\
\left\{\bar{\theta} \mathrm{B}^{*}(\tau \lambda)\left[\tau \delta-\bar{\tau} \mathrm{m}_{1}\right]+\tau \mathrm{k}_{1}(\bar{\theta}-\bar{\kappa})\right\}+2 \mathrm{~m}_{1}\left(1-\mathrm{k}_{1}\right)- \\
2 \mathrm{~m}_{1} \mathrm{~B}^{*}(\tau \lambda)[\bar{\tau} \delta+\tau]-2 \tau \mathrm{k}_{1} \bar{\delta} \\
\mathrm{k}_{1}=\lim _{z \rightarrow 1} \frac{\mathrm{d}}{\mathrm{dz}} \mathrm{B}^{*}(\lambda-\bar{\tau} \lambda \mathrm{c}(\mathrm{z}))
\end{aligned}
$$

The average system size is given by

$$
\begin{aligned}
\mathrm{L}_{S} & =\lim _{z \rightarrow 1} \frac{\mathrm{d}}{\mathrm{dz}} \mathrm{P}_{S}(\mathrm{z}) \\
& =\mathrm{L}_{\mathrm{q}}+\mathrm{S}
\end{aligned}
$$

\section{SPECIAL CASES}

Case (i)

If $\tau=0, \theta=0$ (no priority, no collision, no orbital search) then the model reduce to single phase $\mathrm{M} / \mathrm{G} / 1$ retrial queue with feedback. In this case

$$
\begin{gathered}
R(\mathrm{z})=\mathrm{R}_{0}\left(1-\mathrm{A}^{*}(\lambda)\right)[\mathrm{z}-\mathrm{c}(\mathrm{z})(\bar{\delta}+\delta \mathrm{z}) \\
\left.\mathrm{B}^{*}(\lambda-\lambda \mathrm{c}(\mathrm{z}))\right] / \mathrm{D}(\mathrm{z}) \\
\mathrm{S}(\mathrm{z})=R_{0} \mathrm{~A}^{*}(\lambda)\left[1-\mathrm{B}^{*}(\lambda-\lambda \mathrm{c}(\mathrm{z}))\right] / \mathrm{D}(\mathrm{z})
\end{gathered}
$$




$$
\begin{gathered}
\mathrm{P}_{\mathrm{s}}(\mathrm{z})=\mathrm{R}_{0} \mathrm{~A}^{*}(\lambda) \mathrm{B}^{*}(\lambda-\lambda \mathrm{c}(\mathrm{z}))(1-\mathrm{z}) \bar{\delta} / \mathrm{D}(\mathrm{z}) \\
\mathrm{P}_{\mathrm{q}}(\mathrm{z})=\mathrm{R}_{0} \mathrm{~A}^{*}(\lambda)[1-\mathrm{z}]\left(1-\delta \mathrm{B}^{*}(\lambda-\bar{\tau} \lambda \mathrm{c}(\mathrm{z}))\right) / \mathrm{D}(\mathrm{z}) \\
\mathrm{D}(\mathrm{z})=\left[\mathrm{c}(\mathrm{z})+(1-\mathrm{c}(\mathrm{z})) \mathrm{A}^{*}(\lambda)\right](\bar{\delta}+\delta \mathrm{z}) \\
\mathrm{B}^{*}(\lambda-\lambda \mathrm{c}(\mathrm{z}))-\mathrm{z}
\end{gathered}
$$

The above result agree with the result of Sumitha and Udaya Chandrika [2011] with $\beta=\theta=0$.

\section{Case (ii)}

Suppose that $\mathrm{C}(\mathrm{z}) \rightarrow \mathrm{z}, \delta=0, \mathrm{\kappa}=1, \theta=0$ (Single arrival, no feedback, no collision, no orbital search) then the model reduces to sole influx retrial queueing classical with preemptive recommence importance. In this case

$$
\begin{aligned}
& R(\mathrm{z})= R_{0}\left(1-\mathrm{A}^{*}(\lambda)\right)\left(1-\mathrm{B}^{*}(\lambda-\bar{\tau} \lambda \mathrm{z})\right) \\
& \mathrm{z}(1-\mathrm{z}) / \mathrm{D}(\mathrm{z}) \\
& \mathrm{S}(\mathrm{z})=\mathrm{R}_{0}(1-\mathrm{z}) \mathrm{A}^{*}(\lambda)\left[1-\mathrm{B}^{*}(\lambda-\bar{\tau} \lambda \mathrm{z})\right] / \mathrm{D}(\mathrm{z}) \\
& \mathrm{P}_{\mathrm{S}}(\mathrm{z})=\mathrm{R}_{0} \mathrm{~A}^{*}(\lambda)(1-\bar{\tau} \mathrm{z}) \mathrm{B}^{*}(\lambda-\bar{\tau} \lambda \mathrm{z})(1-\mathrm{z}) / \mathrm{D}(\mathrm{z}) \\
& \mathrm{P}_{\mathrm{q}}(\mathrm{z})=\mathrm{R}_{0} \mathrm{~A}^{*}(\lambda)(1-\mathrm{z})\left(1-\mathrm{z}+\mathrm{z} \tau \mathrm{B}^{*}(\lambda-\bar{\tau} \lambda \mathrm{z})\right) \\
& \quad / \mathrm{D}(\mathrm{z}) \\
& \mathrm{D}(\mathrm{z})=\mathrm{B}^{*}(\lambda-\bar{\tau} \lambda \mathrm{z})\left\{(1-\bar{\tau} \mathrm{z})\left[\mathrm{z}+(1-\mathrm{z}) \mathrm{A}^{*}(\lambda)\right]\right. \\
&\left.-\tau \mathrm{z}^{2}\right\}-\mathrm{z}(1-\mathrm{z})
\end{aligned}
$$

The above results agree with the result of Krishna Kumar et al.[2002] with $\mathrm{p}=0$.

\section{Stochastic Decomposition}

on this segment, we speak the Stochastic decomposition stuff of the system size circulation. From the rottenness belongings the probability producing characteristic of the device size distribution may be disintegrated as $\mathrm{ps}(\mathrm{z})=\Pi(\mathrm{z}) \times \Psi(\mathrm{z})$ where $\Pi(\mathrm{z})$ is the opportunity generating characteristic of the number of clients within the batch onset column with precedence, collisions, remarks and orbital seek and $\Psi(\mathrm{z})$ be the possibility producing feature of the quantity of clients inside the path while the machine is idle. 


$$
\begin{aligned}
& \quad \Pi(\mathrm{z})=\frac{\left(\tau \mathrm{B}^{*}(\tau \lambda)-\delta \tau \mathrm{B}^{*}(\tau \lambda)-\mathrm{m}_{1}\left(1-\mathrm{B}^{*}(\tau \lambda)\right)\right)(1-\mathrm{z})(1-\bar{\tau} \mathrm{c}(\mathrm{z}))}{\tau\left\{\left(\mathrm{zc}(\mathrm{z})-\tau \mathrm{zc}(\mathrm{z}) \mathrm{B}^{*}(\tau \lambda)-\mathrm{z}+\mathrm{B}^{*}(\tau \lambda)(\delta \mathrm{z}+\bar{\delta})(1-\bar{\tau} \mathrm{c}(\mathrm{z}))\right)\right\}} \\
& \Psi(\mathrm{z})=\frac{\mathrm{R}_{0}+\mathrm{R}(\mathrm{z})}{\mathrm{R}_{0}+\mathrm{R}} \\
& =\frac{\left\{\left(\mathrm{zc}(\mathrm{z})-\tau \mathrm{zc}(\mathrm{z}) \mathrm{B}^{*}(\tau \lambda)-\mathrm{z}+\mathrm{B}^{*}(\tau \lambda)(\delta \mathrm{z}+\bar{\delta})(1-\bar{\tau} \mathrm{c}(\mathrm{z}))\right)\right\} \mathrm{x}}{\mathrm{D}(\mathrm{z})\left(\tau \mathrm{B}^{*}(\tau \lambda)-\delta \tau \mathrm{B}^{*}(\tau \lambda)-\mathrm{m}_{1}\left(1-\mathrm{B}^{*}(\tau \lambda)\right)\right)} \\
& \text { where } \mathrm{X}=\tau \mathrm{R}_{0} \mathrm{~A}^{*}(\lambda) \mathrm{B}^{*}(\lambda-\bar{\tau} \lambda \mathrm{c}(\mathrm{z})) \bar{\delta}
\end{aligned}
$$

\section{Numerical Results}

In this segment, we achieve some arithmetical outcomes the usage of MATLAB. assume that retrial time, provider times are exponentially disbursed with price $\eta$ and $\mu$. We pick a few arbitrary values for the parameters $\lambda=2 ; \theta=0.3 ; \mathrm{c} 1=$ zero. $5 ; \mathrm{c} 2=0.5 ; \tau=0.3 ; \kappa=0.3 ; \eta=10 ; \delta=0.2$; $\mu=20$; The effect of various parameters at the enactment measure R 0 -the opportunity that the machine is blank, R- the probability that the server is idle in the non-empty machine and Sthe chance that the servers is hectic are calculated and outcomes are offered in the table 1 and 2.

Table 1 shows that

- $\quad$ R0 proliferations with upsurge in $\mu$ and $\eta$ and reductions with upsurge in $\lambda$

- $\quad$ R reductions with upsurge in $\eta$ and $\mu$ and proliferations with upsurge in $\lambda$

- $\quad$ S upsurges with proliferation in $\lambda$, reductions with upsurge in $\mu$ and autonomous of $\eta$

Table 2 presents that

- $\quad$ R0 upsurges with proliferation in $\theta$ and $\kappa$ and reductions with upsurge in $\tau$

- $\quad$ R reductions with upsurge in $\theta$ and $\kappa$ and proliferations with upsurge in $\tau$

- $\quad \mathrm{S}$ is independent of $\theta, \tau$ and $\kappa$

Table 1 Performance measures versus $\lambda, \mu, \eta$

\begin{tabular}{|c|c|c|c|c|c|}
\hline $\boldsymbol{\lambda}$ & $\boldsymbol{\mu}$ & $\boldsymbol{\eta}$ & $\mathbf{R}_{\mathbf{0}}$ & $\mathbf{R}$ & $\mathbf{S}$ \\
\hline \multirow{4}{*}{2} & & 4 & 0.4425 & 0.3075 & 0.2500 \\
\cline { 3 - 6 } & \multirow{3}{*}{15} & 8 & 0.5963 & 0.1537 & 0.2500 \\
\cline { 3 - 6 } & & 12 & 0.6475 & 0.1025 & 0.2500 \\
\cline { 3 - 6 } & \multirow{3}{*}{20} & 16 & 0.6731 & 0.0769 & 0.2500 \\
\cline { 3 - 6 } & & 4 & 0.5428 & 0.2697 & 0.1875 \\
\cline { 3 - 6 } & & 8 & 0.6777 & 0.1348 & 0.1875 \\
\cline { 3 - 6 } & & 12 & 0.7226 & 0.0899 & 0.1875 \\
\hline
\end{tabular}




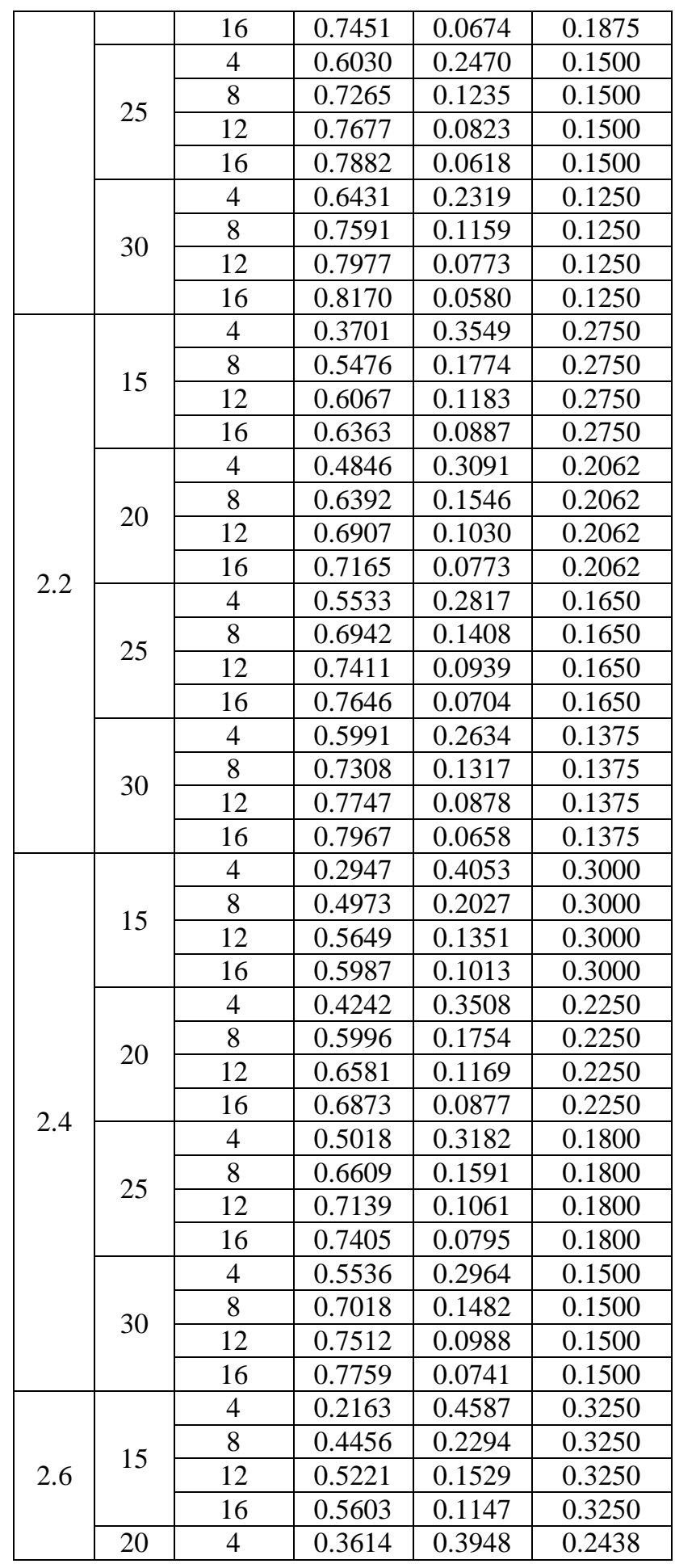




\begin{tabular}{|c|c|c|c|c|}
\hline & 8 & 0.5588 & 0.1974 & 0.2438 \\
\hline & 12 & 0.6246 & 0.1316 & 0.2438 \\
\hline & 16 & 0.6575 & 0.0987 & 0.2438 \\
\hline \multirow{4}{*}{25} & 4 & 0.4485 & 0.3565 & 0.1950 \\
\hline & 8 & 0.6268 & 0.1782 & 0.1950 \\
\hline & 12 & 0.6862 & 0.1188 & 0.1950 \\
\hline & 16 & 0.7159 & 0.0891 & 0.1950 \\
\hline \multirow{4}{*}{30} & 4 & 0.5066 & 0.3309 & 0.1625 \\
\hline & 8 & 0.6720 & 0.1655 & 0.1625 \\
\hline & 12 & 0.7272 & 0.1103 & 0.1625 \\
\hline & 16 & 0.7548 & 0.0827 & 0.1625 \\
\hline
\end{tabular}

Table 2 Performance measures versus $\theta, \tau, \kappa$

\begin{tabular}{|c|c|c|c|c|c|}
\hline$\theta$ & $\tau$ & k & $\mathbf{R}_{\mathbf{0}}$ & $\mathbf{R}$ & $\mathbf{S}$ \\
\hline \multirow{16}{*}{0.1} & \multirow{4}{*}{0.4} & 0.30 & 0.6270 & 0.1855 & 0.1875 \\
\hline & & 0.45 & 0.6292 & 0.1833 & 0.1875 \\
\hline & & 0.60 & 0.6315 & 0.1810 & 0.1875 \\
\hline & & 0.75 & 0.6337 & 0.1788 & 0.1875 \\
\hline & \multirow{4}{*}{0.5} & 0.30 & 0.6244 & 0.1881 & 0.1875 \\
\hline & & 0.45 & 0.6272 & 0.1853 & 0.1875 \\
\hline & & 0.60 & 0.6300 & 0.1825 & 0.1875 \\
\hline & & 0.75 & 0.6328 & 0.1797 & 0.1875 \\
\hline & \multirow{4}{*}{0.6} & 0.30 & 0.6218 & 0.1907 & 0.1875 \\
\hline & & 0.45 & 0.6251 & 0.1874 & 0.1875 \\
\hline & & 0.60 & 0.6285 & 0.1840 & 0.1875 \\
\hline & & 0.75 & 0.6319 & 0.1806 & 0.1875 \\
\hline & \multirow[b]{4}{*}{0.7} & 0.30 & 0.6191 & 0.1934 & 0.1875 \\
\hline & & 0.45 & 0.6231 & 0.1894 & 0.1875 \\
\hline & & 0.60 & 0.6270 & 0.1855 & 0.1875 \\
\hline & & 0.75 & 0.6309 & 0.1816 & 0.1875 \\
\hline \multirow{11}{*}{0.2} & \multirow{4}{*}{0.4} & 0.30 & 0.6645 & 0.1480 & 0.1875 \\
\hline & & 0.45 & 0.6667 & 0.1458 & 0.1875 \\
\hline & & 0.60 & 0.6690 & 0.1435 & 0.1875 \\
\hline & & 0.75 & 0.6712 & 0.1413 & 0.1875 \\
\hline & \multirow{4}{*}{0.5} & 0.30 & 0.6619 & 0.1506 & 0.1875 \\
\hline & & 0.45 & 0.6647 & 0.1478 & 0.1875 \\
\hline & & 0.60 & 0.6675 & 0.1450 & 0.1875 \\
\hline & & 0.75 & 0.6703 & 0.1422 & 0.1875 \\
\hline & \multirow{3}{*}{0.6} & 0.30 & 0.6593 & 0.1532 & 0.1875 \\
\hline & & 0.45 & 0.6626 & 0.1499 & 0.1875 \\
\hline & & 0.60 & 0.6660 & 0.1465 & 0.1875 \\
\hline
\end{tabular}




\begin{tabular}{|c|c|c|c|c|c|}
\hline & & 0.75 & 0.6694 & 0.1431 & 0.1875 \\
\hline & \multirow[b]{4}{*}{0.7} & 0.30 & 0.6566 & 0.1559 & 0.1875 \\
\hline & & 0.45 & 0.6606 & 0.1519 & 0.1875 \\
\hline & & 0.60 & 0.6645 & 0.1480 & 0.1875 \\
\hline & & 0.75 & 0.6684 & 0.1441 & 0.1875 \\
\hline \multirow{16}{*}{0.3} & \multirow{4}{*}{0.4} & 0.30 & 0.7020 & 0.1105 & 0.1875 \\
\hline & & 0.45 & 0.7042 & 0.1083 & 0.1875 \\
\hline & & 0.60 & 0.7065 & 0.1060 & 0.1875 \\
\hline & & 0.75 & 0.7087 & 0.1037 & 0.1875 \\
\hline & \multirow{4}{*}{0.5} & 0.30 & 0.6994 & 0.1131 & 0.1875 \\
\hline & & 0.45 & 0.7022 & 0.1103 & 0.1875 \\
\hline & & 0.60 & 0.7050 & 0.1075 & 0.1875 \\
\hline & & 0.75 & 0.7078 & 0.1047 & 0.1875 \\
\hline & \multirow{4}{*}{0.6} & 0.30 & 0.6968 & 0.1157 & 0.1875 \\
\hline & & 0.45 & 0.7001 & 0.1124 & 0.1875 \\
\hline & & 0.60 & 0.7035 & 0.1090 & 0.1875 \\
\hline & & 0.75 & 0.7069 & 0.1056 & 0.1875 \\
\hline & \multirow[b]{4}{*}{0.7} & 0.30 & 0.6941 & 0.1184 & 0.1875 \\
\hline & & 0.45 & 0.6981 & 0.1144 & 0.1875 \\
\hline & & 0.60 & 0.7020 & 0.1105 & 0.1875 \\
\hline & & 0.75 & 0.7059 & 0.1066 & 0.1875 \\
\hline \multirow{16}{*}{0.4} & \multirow{4}{*}{0.4} & 0.30 & 0.7395 & 0.0730 & 0.1875 \\
\hline & & 0.45 & 0.7417 & 0.0708 & 0.1875 \\
\hline & & 0.60 & 0.7440 & 0.0685 & 0.1875 \\
\hline & & 0.75 & 0.7462 & 0.0663 & 0.1875 \\
\hline & \multirow{4}{*}{0.5} & 0.30 & 0.7369 & 0.0756 & 0.1875 \\
\hline & & 0.45 & 0.7397 & 0.0728 & 0.1875 \\
\hline & & 0.60 & 0.7425 & 0.0700 & 0.1875 \\
\hline & & 0.75 & 0.7453 & 0.0672 & 0.1875 \\
\hline & \multirow{4}{*}{0.6} & 0.30 & 0.7343 & 0.0782 & 0.1875 \\
\hline & & 0.45 & 0.7376 & 0.0749 & 0.1875 \\
\hline & & 0.60 & 0.7410 & 0.0715 & 0.1875 \\
\hline & & 0.75 & 0.7444 & 0.0681 & 0.1875 \\
\hline & \multirow[b]{4}{*}{0.7} & 0.30 & 0.7316 & 0.0809 & 0.1875 \\
\hline & & 0.45 & 0.7356 & 0.0769 & 0.1875 \\
\hline & & 0.60 & 0.7395 & 0.0730 & 0.1875 \\
\hline & & 0.75 & 0.7434 & 0.0691 & 0.1875 \\
\hline
\end{tabular}

\section{Conclusion}

In these articles, we explored consignment advent retrial queueing version with preemptive recommence importance, collisions, remarks and orbital search. Constant kingdom 
equations, possibility producing characteristic for the numbers of clients in the machine and in the queue are determined by means of additional adjustable approach. Enactment measure like likelihood of the idle servers, probability of the busy server, average orbit length and average gadget size are computed. unique cases for this version are deliberated. The impact of the limitations on the enactment measure is computed mathematically. Our recommended version has capability practical real existence application in optical burst switching community to ahead the packets inside a network for transmission. Different actual life programs are call centres, random get admission to protocols, mobile networks, software designs and manufacturing device and so on. The overall decay rule has been recognized for this classical. In future, this model can be further extended with many features like, vacation, breakdown, optional service, impatient customers etc. And we can find cost optimization for this prescribed model.

\section{References}

[1] Ayyapan, G. and Udaya Geetha, J. “Analysis of pre-emptive priority retrial queueuing system with starting failure, modified bernoulli vacation, with vacation interruption, repair immediate feedback and impatient customers", International Journal of Mathematics and its Applications, vol.6, (1-E), pg. 1013-1023, 2018.

[2] Chakravarthy, S.R., Krishnamoorthy, A. and Joshua,V.C. "Analysis of a multi-server retrial queue with search of customers from the orbit", Performance Evaluation, vol. 63, no.8, pg.776-798, 2006.

[3] Deepak, T.G., Dudin, A.N., Joshua, V.C and krishnamoorthy, A. "On an Mx/G/1 retrial system with two types of search of customers from the orbit", Stochastic Analysis and Applications", vol.31, no. 1, pg. 92-107, 2012.

[4] Dimitriou, I. " A pre-emptive resume priority retrial queue with state dependent arrivals, unreliable server and negative customers", TOP , 21, pg. 542-571, 2013.

[5] Gao, S. and Wang, J., "Performance and reliability analysis of an M/G/1 - G retrial queue with orbital search and non persistent customers", European Journal of Operation Research, vol.236, pg. 561-572, 2014.

[6] Jain, M. and Charu Bhargava , "Bulk arrival retrial queue with unreliable server and priority subscribers”, International Journal of Operations Research, vol. 5, no. 4, pg. 242-259, 2008.

[7] Juntong $\mathrm{Li}$, Jinping $\mathrm{Xu}$ and $\mathrm{Tao} \mathrm{Li}$, “ $\mathrm{An} \mathrm{M} / \mathrm{M} / 1$ retrial queue with working vacation and collision", International Journal of Research and Reviews in Applied Sciences, vol. 39, no.1, pg. 40-46, 2019.

[8] Ke , J.C and Chang, F.M, “ Modified vacation policy for M/G/1 retrial queue with balking and feedback”, Computational Industrial Engineering, vol. 57, pg. 433-443, 2009.

[9] Krishnakumar, B., Vijayakumar, A. and Arivaudainambi, D. "An M/G/1 retrial queueing system with two phase service and preemptive resume", Annals of Operation Research, vol. 113, pg. $61-79,2002$.

[10] Krishnakumar, B., Vijayalakshmi, G., Krishnamoorthy, A., and Sadiq Basha, S., “ A Single server feedback retrial queue with collisions", Computers and Operations Research, vol. 37, pg.1247-1255, 2010.

[11] Kvach , A. and Nazarov, A. " Sejourn time analysis of finite source markov retrial queueing system with collisions", Proceedings of 14 th International Scientific Conference, pg. 18-22, 2015.

[12] Lakshmi, K. and Ramanath, K. "An M/G/1 two phase multi optional retrial queue with Bernoulli feedback, non-persistent customers and breakdown and repair”, International Journal of Operational Research, vol. 19, no. 1, pg. 78-95, 2014. 
[13] Liu, z., Peng, Y. and Wu, J. “ An M/G/1 retrial G-queue with pre-emptive resume priority and collisions subject to the server breakdown and delayed repairs", Journal of Applied Mathematics and Computing, vol 44, no. 2, pg. 187-213, 2014.

[14] Meer, H.D. “ Finite source M/M/S retrial queue with search for balking and impatient customers from the orbit”, Computer Networks, vol 53, no. 8, pg. 1264-1273, 2009.

[15] Murugan, P.B., and Vijaya Krishnaraj, R., " A bulk arrival retrial queue with orbital search and exponentially distributed multiple working vacation”, AIP Conference Proceedings, vol. 2177, doi.org/10.1063/1.5135240, 2019.

[16] Peishu Chen, Yongwu Zhou and Changwen Li , " Batch arrival retrial G- queue with orbital search and non persistent customers", Journal of Interdisciplinary Mathematics, vol 19, no. 1, pg. 95-109, 2016.

[17] Peng, Y. "On the discrete time Geo/G/1 retrial queueing system with pre-emptive resume and Bernoulli feedback", Opsearch, vol.53, no.1, pg. 116-130, 2016.

[18] Rajadurai, P., Chandrasekaran, V.M. and Saravanarajan, M.C," Steady state analysis of batch arrival feedback retrial queue with two phases of service, negative customers, bernoulli vacation, and server breakdown", International Journal of Mathematical Operations Research, vol. 7, pg. 519-546, 2015.

[19] Peishu Chen, Yijuan Zhu, “An M/G/1 retrial queue with priority, balking and feedback customers", Journal of Convergence Information Technology, vol 5, no. 02, pg. 155-162, 2010.

[20] Sherif Ammar, I. and Rajadurai, P. " Performance analysis of pre-emptive priority retrial queueing system with disaster under working breakdown services", Symmetry MDPI, vol 11, no 419, pg. 01-15, DOI. 10.3390, 2019.

[21] Sumitha,D. and Udaya Chandrika, K. "Performance measures of feedback batch arrival retrial queue with optional second service and orbital search", Advances in Applied Research, vol. 3, no. 2, pg. 179-186, 2011.

[22] Tao, L., Liu, Z. and Wang, Z.," M/M/1 retrial queue with collisions and working vacation interruption under N policy", RAIRO Operations Research, vol. 46, pg. 355-371, 2012.

[23] Toth ,A. ,Berczes, T., Sztrik, J.,Kvach, A.” Simulation of finite source retrial queueing system with collisions and non reliable server", International Conference on distributed Computer and Communication networks, pg. 146-158, 2017.

[24] Varalakshmi, M., Chandrasekaran, V.M. and Saravanarajan, M.C.,"An unreliable two phase retrial queue with immediate Bernoulli feedbacks", International Journal of Pure and Applied Mathematics, vol. 115, no. 9, pg. 447-457, 2017. 